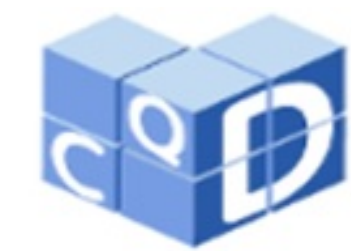

Revista Eletrônica

Paulista de Matemática

ISSN 2316-9664

Volume 10, dez. 2017

Edição Ermac

João Paulo de Freitas Gama

Universidade Federal do Rio

Grande do Norte

defreitasgama11@gmail.com

Fabiana Tristão Santana

Universidade Federal do Rio

Grande do Norte

fabianatsantana@gmail.com

\section{Utilização do software GeoGebra para aproximar funções através de mínimos quadrados}

Using GeoGebra software to approximate functions through least squares

\section{Resumo}

Para este trabalho foi feito um estudo detalhado de aproximações por Mínimos Quadrados em espaços vetoriais ortogonais. Em particular, utilizamos esta teoria para obter a melhor aproximação de uma função $f$ no espaço $W$, gerado pelas funções $1, \cos (t), \cos (2 t), \ldots, \cos (n t), \operatorname{sen}(t), \operatorname{sen}(2 t), \ldots$, e $\operatorname{sen}(n t)$ utilizando um produto interno apropriado. Tal função é denominada de polinômio trigonométrico e coincide com a n-ésima soma parcial da série de Fourier da função $f$, uma vez que seus coeficientes coincidem com os coeficientes de Fourier de $f$. O polinômio obtido foi utilizado para aproximar uma força que atua sobre um sistema mecânico com o uso do software GeoGebra o que permitiu compreender melhor a construção da função e, ao mesmo tempo, a representação gráfica mostrou que aumentando o grau do polinômio melhor é a aproximação obtida.

Palavras-chave: Mínimos quadrados. Séries de Fourier. Sistemas mecânicos. GeoGebra. Matemática aplicada à física

\begin{abstract}
For this work a detailed study of approximations by least squares in orthogonal vector spaces. In particular, we use this theory to get the best approximation of a function $f$ in space $W$ generated by functions $1, \cos (t), \cos (2 t), \ldots, \cos (n t), \operatorname{sen}(t), \operatorname{sen}(2 t), \ldots, \operatorname{sen}(n t), \quad \ldots$, using an appropriate internal product. This function is called a trigonometric polynomial and coincides with the nth partial sum of the Fourier series of the function $f$, once their coefficients coincide with the Fourier coefficients of $f$. The obtained polynomial was used to approximate a force that acts on a mechanical system with the use of the software GeoGebra which allowed to better understand the construction of the function and, at the same time, the graphic representation showed that increasing the degree of the polynomial better is the approximation Obtained.
\end{abstract}

Keywords: Least squares. Fourier series. Mechanical systems. GeoGebra. Mathematics applied to physics. 


\section{Introdução}

Sistema de equações lineares, ou simplemente sistema linear, constitue um importante tópico da Álgebra Linear com aplicações em diversas áreas, como Física, Química e Engenharias. Em geral, representa-se um sistema linear pela equação $A \vec{x}=\vec{b}$, onde $\vec{x}$ é o vetor coluna de $n$ linhas cujas coordenadas são as variáveis do sistema, $A$ é uma matriz de $m$ linhas e $n$ colunas cujo termo geral $a_{i j}$ é o coeficiente da j-ésima variável localizada na i-ésima equação e, por fim, $\vec{b}$ é um vetor coluna de $m$ linhas cujas entradas são os termos independentes do sistema.

Se $W$ é o subespaço gerado pelos vetores coluna de $A$, denotados por $\overrightarrow{c_{1}}, \overrightarrow{c_{2}}, \ldots \overrightarrow{c_{n}}$, a existência de solução para $A \vec{x}=\vec{b}$ indica que $\vec{b} \in W$, isto é, existem escalares $\alpha_{1}, \alpha_{2}, \ldots, \alpha_{n}$ satisfazendo a equação $\alpha_{1} \overrightarrow{c_{1}}+\alpha_{2} \overrightarrow{c_{2}}+\ldots+\alpha_{n} \overrightarrow{c_{n}}=\vec{b}$.

Os sistemas lineares estão presentes em muitas aplicações e é comum que erros de medições, aproximações feitas durante a modelagem e, até mesmo, a limitação do sistema computacional tragam imprecisões aos coeficientes das variáveis a ponto de tornar o sistema inconsistente e, com isso, o vetor $\vec{b}$ não pertence mais a $W$. Nesses casos, onde o sistema algebricamente é inconsistente, a Álgebra Linear fornece a melhor solução aproximada através do processo de mínimos quadrados, que consiste em substituir o vetor $\vec{b}$ pelo vetor de $W$ que mais se aproxima dele.

Com a técnica dos mínimos quadrados, a melhor aproximação em $W$ para o vetor $\vec{b}$ é a projeção ortogonal de $\vec{b}$ em $W$, obtida utilizando um produto interno apropriado e resultados importantes da Álgebra Linear.

Em particular, ao considerar o espaço das funções reais com um produto interno apropriado e $W$ sendo o subespaço gerado por funções trigonométricas ortogonais, a aproximação da função real $f$ em $W$ dá origem a uma função conhecida por polinômio trigonométrico que coincide com a n-ésima soma parcial da série de Fourier de $f$. As séries de Fourier, são séries infinitas utilizadas para representar funções periódicas gerais em termos de senos e cossenos, desempenham um importante papel nas aplicações da engenharia e tem muita importâcia na solução de EDOs e EDPs, bem como na análise de sistemas mecânicos e elétricos sob a ação de estímulos externos e periódicos.

Este trabalho tem como objetivo utilizar os conceitos da Álgebra Linear, como produto interno, projeção ortogonal e processo de mínimos quadrados, para deduzir a melhor aproximação para uma função real $f$ no espaço das funções periódicas gerado por funções trigonométricas ortogonais. Será apresentada uma aplicação, modelada por um sistema mecânico, que mostra a necessidade da aproximação de funções na resolução de equações diferenciais. Por fim, será utilizado o software GeoGebra para mostrar a eficiência de se agregar ferramentas computacionais para obter aproximação de funções e, ao mesmo tempo, para analisar geometricamente como o grau do polinômio trigonométrico melhora a aproximação obtida. Esta abordagem é uma tentativa de explicar melhor o que foi feito de forma resumida em (GAMA; SANTANA, 2017), principalmente no que se refere à utilização do software GeoGebra na aproximação de funções.

\section{Aproximação de funções por polinômios trigonométricos}

Aproximar funções $f: \mathbb{R} \rightarrow \mathbb{R}$ contínuas em $[-L, L]$ por outras constituídas por somas de senos e cossenos, como $F(t)=a_{0}+a_{1}+\cos \left(\frac{\pi t}{L}\right)+a_{2} \cos \left(\frac{2 \pi t}{L}\right)+\ldots+a_{n} \cos \left(\frac{n \pi t}{L}\right)+b_{1} \operatorname{sen}\left(\frac{\pi t}{L}\right)$ 
$+b_{2} \operatorname{sen}\left(\frac{2 \pi t}{L}\right)+\ldots+b_{n} \operatorname{sen}\left(\frac{n \pi t}{L}\right)$, é frequentemente usado para facilitar a resolução de equações diferenciais, como é ressaltado em (ZILL; CULLEN, 2007).

Considerando o espaço $W$ gerado pelas funções ortonormais $\phi_{0}(t)=\frac{1}{\sqrt{2 L}}, \phi_{k}(t)=\frac{1}{\sqrt{L}} \cos \frac{k \pi t}{L} \mathrm{e}$ $\varphi_{k}(t)=\frac{1}{\sqrt{L}} \operatorname{sen} \frac{k \pi t}{L}$, para $1 \leq k \leq n$, com o produto interno $\langle g, h\rangle=\int_{-L}^{L} \phi(t) \varphi(t) d t$, com a Álgebra Linear, em particular com o processo de mínimos quadrados, é possível mostrar que a melhor aproximação da função $f$ contínua $[0,2 \pi]$ em $W$ é a projeção $F(t)=\left\langle f, \phi_{0}\right\rangle \phi_{0}+\left\langle f, \phi_{1}\right\rangle \phi_{1}+\ldots+$ $\left\langle f, \phi_{n}\right\rangle \phi_{n}+\left\langle f, \varphi_{1}\right\rangle \varphi_{1}+\left\langle f, \varphi_{2}\right\rangle \varphi_{2}+\ldots+\left\langle f, \varphi_{n}\right\rangle \varphi_{n}$.

Desenvolvendo os cálculos e considerando $a_{0}=\frac{1}{L} \int_{-L}^{L} f(t) d t, a_{n}=\frac{1}{L} \int_{-L}^{L} f(t) \cos \frac{n \pi t}{L} d t$, e $b_{n}=\frac{1}{L} \int_{-L}^{L} f(t) \operatorname{sen} \frac{n \pi t}{L} d t$ obtém-se

$$
F(t)=\frac{a_{0}}{2}+\sum_{k=1}^{n} a_{k} \cos \frac{n \pi t}{L}+\sum_{k=1}^{n} b_{k} \operatorname{sen} \frac{n \pi t}{L}
$$

que coincide com a $n$-ésima soma parcial da série de Fourier da função $f$. Alguns detalhes dos cálculos podem ser vistos em (ANTON; RORRES, 2001) e (ZILL; CULLEN, 2007).

\section{Aplicação}

Esta seção traz uma aplicação que tem por objetivo ressaltar a necessidade e importância das aproximações de funções. A Figura 1 mostra um sistema mecânico estudado em (RAO, 2008) que é conhecido por martelo de forjar. O martelo de forjar consiste em um suporte, um martelo de queda conhecido como pilão (ou martelo-pilão), uma bigorna e um bloco de base. A bigorna é um bloco maciço de aço no qual o material é forjado até a forma desejada pelos golpes repetidos do martelo-pilão. A bigorna normalmente é montada sobre um coxim elástico para reduzir a transmissão de vibração para o bloco de base e suporte.

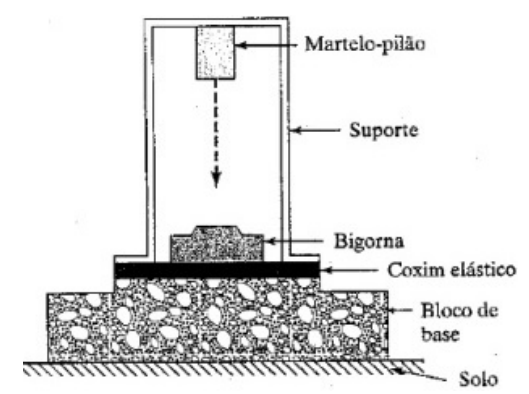

Figura 1: Sistema mecânico conhecido por martelo de forjar.

Modelando este sistema e fazendo algumas considerações obtém-se um sistema massa mola, mostrado na Figura 2, onde a massa está presa na extremidade superior de uma mola cuja extremidade inferior está ligada a um suporte rígido.

De acordo com a segunda lei de Newton, a resultante das forças que atuam sobre um corpo deve ser igual ao produto da massa do corpo por sua aceleração. Considerando a massa do corpo igual a $M, k$ sendo a constante da mola e $x$ sendo o deslocamento em função do tempo, temos que

$$
f(t)-k x=M x^{\prime \prime}
$$




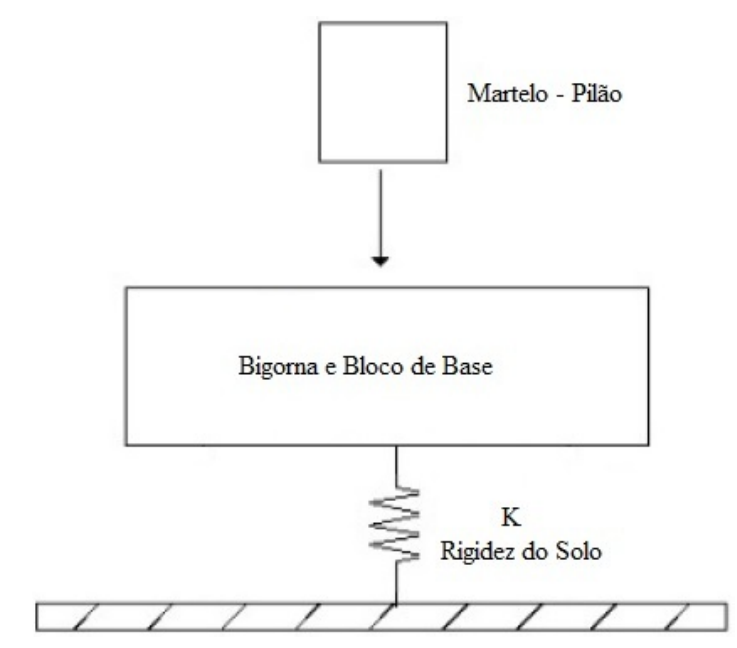

Figura 2: Sistema mecânico simplificado.

isto é

$$
M x "+K x=f(t)
$$

A equação diferencial representada na equação (2) modela muitos problemas e é muito usada nos casos em que a força atuante sobre o sistema é senoidal. A necessidade de aproximar a função $f$ surge quando essa força não é senoidal e, para esses casos, é utilizada equação (1) que fornece a melhor aproximação.

Como exemplo, considere a força que atua no sistema sendo a função $f(t)=t^{2}+\pi$, se $-\pi<t<0$ e $f(t)=-t^{2}+\pi$, se $0 \leq t<\pi$. Os cálculos necessários para a obtenção do polinômio trigonométrico que aproxima $f$ serão feitos no software GeoGebra. Este software, além de garantir precisão e rapidez, possui um efeito dinâmico que será utilizado para mostrar a precisão da aproximação obtida à medida que se aumenta do grau do polinômio.

\subsection{Introdução ao software GeoGebra}

O GeoGebra é um aplicativo de matemática dinâmico criado por Markus Hohenwarter em 2001 na Universität Salzburg para ser utilizado em sala de aula que continua sendo desenvolvido na Florida Atlantic University e possui hoje uma comunidade mundial de usuários, (GEOGEBRA, 2017). O programa permite realizar construções geométricas, inserir funções e alterar todos esses objetos dinamicamente, após a construção estar finalizada. O GeoGebra é capaz de lidar com variáveis para números, pontos, vetores, derivar e integrar funções, e ainda oferecer comandos para se encontrar raízes e pontos extremos de uma função. As principais funções e comandos utilizados no software podem ser consultados em (HOHENWARTER; HOHENWARTER, 2017).

Este software é uma ferramenta computacional mais conhecida na área de Geometria. Porém, ela é muito ampla e pode ser utilizada em várias áreas. Por exemplo, em (MANSO; SANTANA, 2016) utilizou-se o GeoGebra para obter a melhor função que aproximasse um conjunto de pontos dados através dos conceitos de mínimos quandrados. Já em (GAMA; SANTANA, 2017), foi apresentada uma aplicação modelada por um sistema mecânico cuja função atuante no sistema foi aproximada utilizando o software GeoGebra utilizando, para isso, principalmente a Entrada de atribuição de comandos, Janela de Álgebra e a Janela de Visualização. 
Além de dar mais detalhes sobre a aproximação de funções no GeoGebra, o presente trabalho tem por objetivo utilizar a Janela CAS, com a qual o usuário pode salvar a sequência de comandos executados e alterá-los quando quiser.

Para a aplicação, serão utilizadas as Janela de Álgebra, Janela de Visualização e a Janela CAS do GeoGebra, como ilustrado na Figura 3. A Janela CAS, pouco menos explorada, é uma excelente ferramenta que consiste em um ambiente dividido em células que interagem entre si onde pode-se realizar operações algébricas envolvendo diversas funções, das mais simples às mais complexas. Esta ferramenta permite ao usuário fazer uma espécie de programação e gravar os passos executados, os quais podem sofrer edições e atualizações instantaneamente em qualquer etapa. É comum alguma função ou comando sofrerem pequenas alterações em suas sintaxes quando utilizadas na Janela CAS ou diretamente na Entrada do GeoGebra. Para mais detalhes sobre as sintaxes dos comandos pode-se consultar (HOHENWARTER; HOHENWARTER, 2017) e (TRINDADE; GREGÓRIO, 2017).

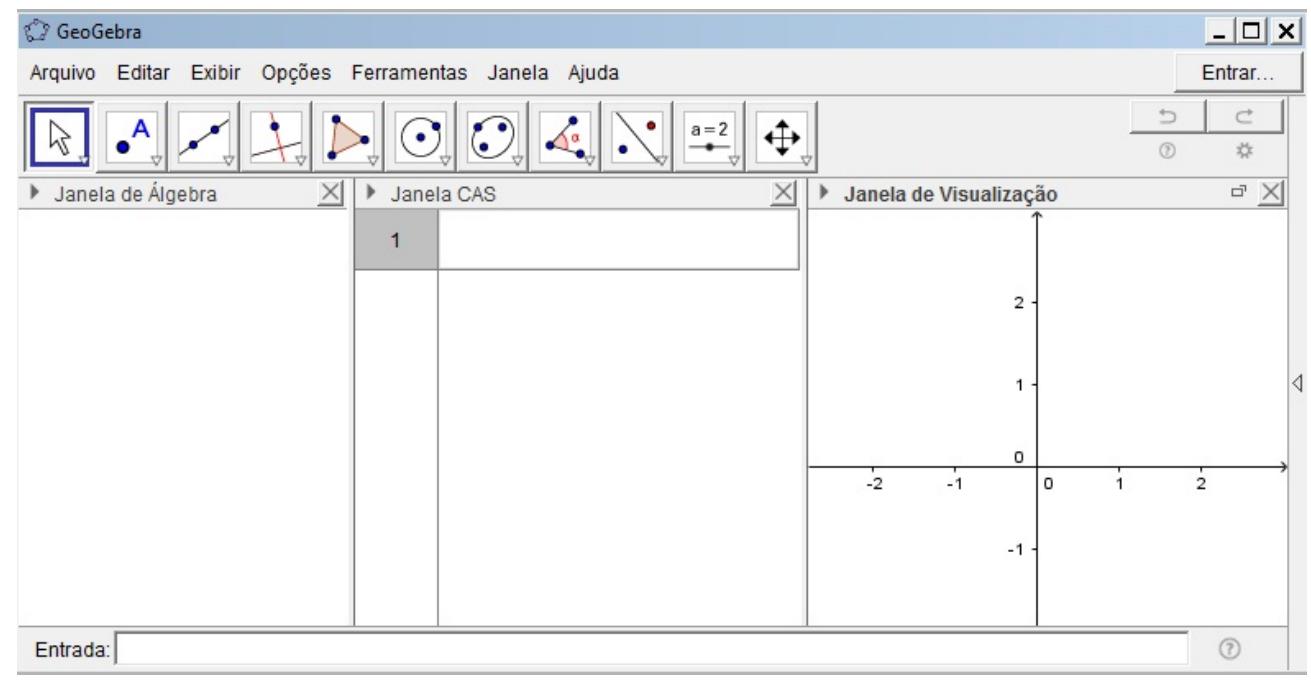

Figura 3: Apresentação do GeoGebra.

As principais funções ou comandos que serão utilizados na Janela CAS do GeoGebra para a dedução do polinômio trigonométrico que aproxima $f$ são "Se $[<$ Condição $>,<$ Então $>$ ]", que define funções com mais de uma sentença, "Efeito Dinâmico", que será usado para dar flexibilidade ao grau do polinômio aproximador, "Integral $[<$ Função $>,<$ Variável $>,<$ Valor Inicial $>,<$ Valor Final $>$ ", para integração e, "Soma[Sequência[Expressão, $<$ Variável $>,<$ Valor Incial $>,<$ Valor Final $>$ ]]", para somar os termos de uma sequência obtidos substituindo a variável pelos valores compreendidos entre "Valor Inicial" e "Valor Final".

\subsection{Aproximação de função utilizando o software GeoGebra}

Aqui serão utilizadas as funções citadas acima para obter o polinômio trigonométrico que aproxima a função $f(t)=t^{2}+\pi$, se $-\pi<t<0$ e $f(t)=-t^{2}+\pi$, se $0 \leq t<\pi$. Para isso, iniciou-se definindo na Célula 1 da Janela CAS do GeoGebra a função $f$ com o comando " $f(x):=S e[-p i<$ $\left.x<0, x^{2}+p i, 0<=x<p i,-x^{2}+p i\right]$ ", como ilustra a Figura 4. Para formatar a função $f$, por exemplo alterar a cor do gráfico, basta ir na Janela de Álgebra e clicar sobre a função com o botão direito do mouse e escolher a opção Propriedades. 


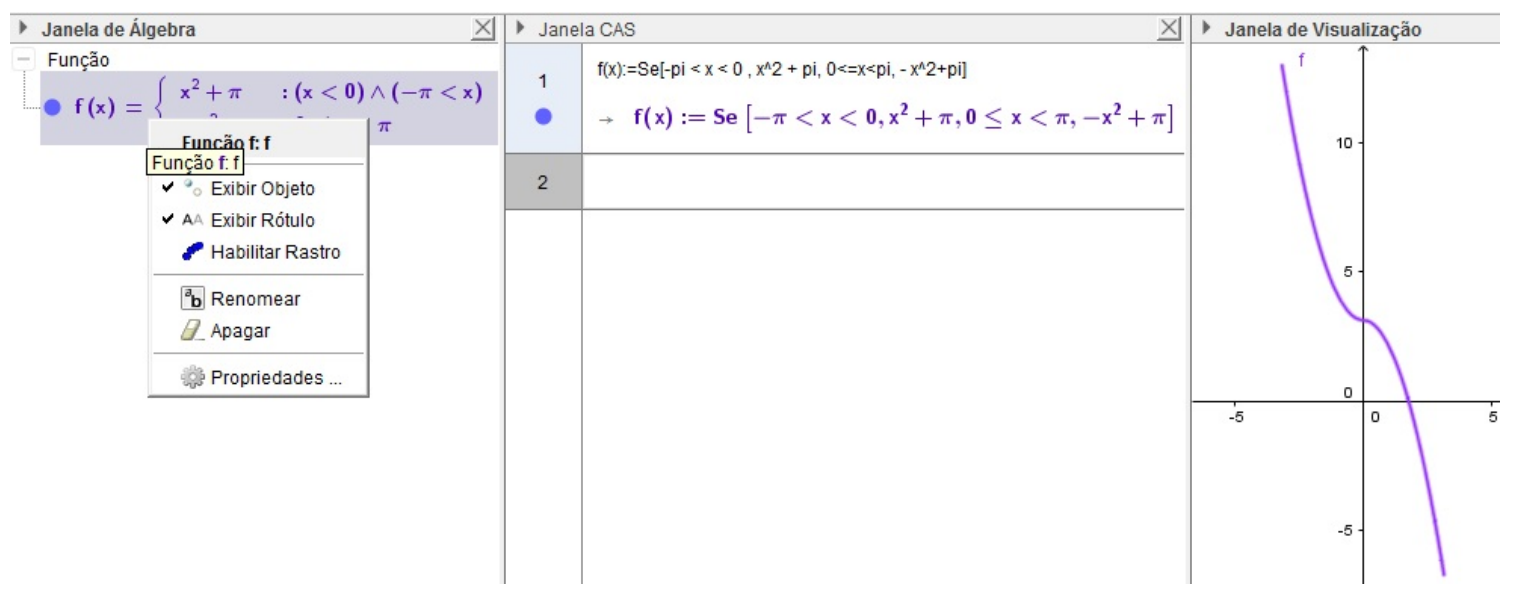

Figura 4: Definição da função $f$.

Em seguida, foi introduzido o efeito dinâmico clicando no botão "Controle Deslizante" e definindo os elementos principais. Foi escolhido o nome $k$ e a variação entre 1 e 15 com um incrento de 1 unidade, que se refere à variação do grau do polinômio. Veja Figura 5.
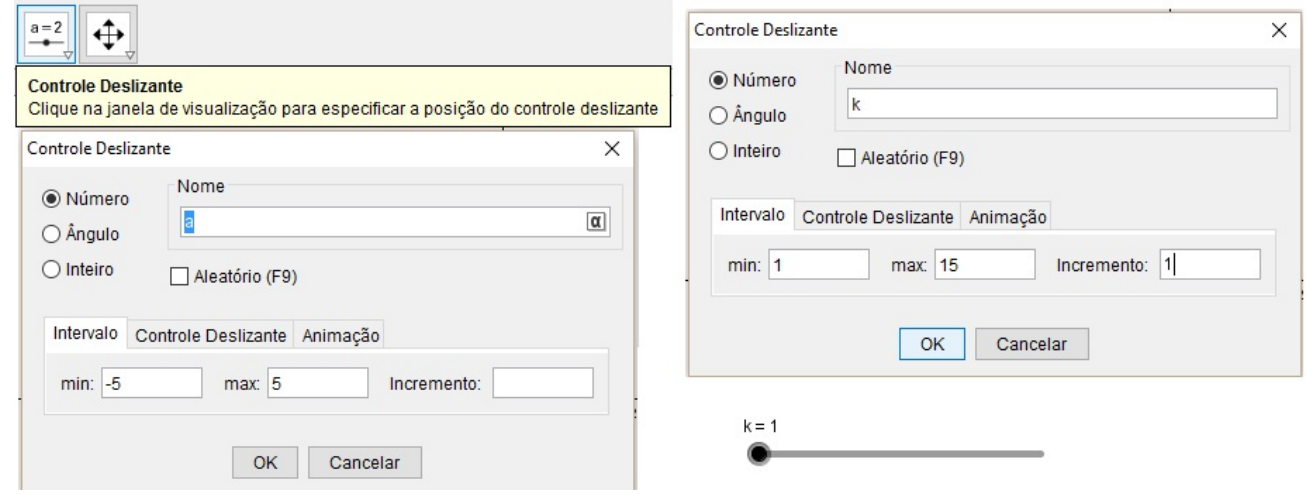

Figura 5: Definição do controle deslizante.

Os coeficientes $a_{0}, a_{k}$ e $b_{k}$, utilizados na equação (1), foram definidos com os comandos "a0:= $(1 /(2 *$ pi $)) *$ Integral[f, x,-pi, pi]", "lista1 = Sequência[ (1/pi) * Integral[f*cos $\left(\left(\mathrm{n}^{*} \mathrm{pi} \mathbf{i}^{*} \mathrm{x}\right) / \mathrm{pi}\right),-\mathrm{pi}$, pi],n,1,k]", "lista2 = Sequência[ (1/pi) * Integral[f*sen $\left(\left(\mathrm{n}^{*} \mathrm{pi}{ }^{*} \mathrm{x}\right) / \mathrm{pi}\right)$, -pi, pi],n,1,k]", respectivamente. $\mathrm{O}$ número de termos de cada lista corresponde ao valor atribuído à $k$. Na Figura 6 pode-se ver a definição dos coeficientes e seus respectivos valores para $k=1$ e $k=3$.

Em seguida, definiu-se os somatórios que compõem o polinômio trigonométrico, definido na equação (1). A soma $G=\sum_{k=1}^{n}\left(a_{k} \cos \frac{k \pi x}{L}\right)$ foi definida com o comando " $\mathrm{g}=$ Soma[Sequência[1/pi * Integral[f*cos((n* $\left.\left.\left.\left.\left.\mathrm{pi}^{*} \mathrm{x}\right) / \mathrm{pi}\right), \mathrm{x},-\mathrm{pi}, \mathrm{pi}\right] * \cos \left(\left(\mathrm{n}^{*} \mathrm{pi}{ }^{*} \mathrm{x}\right) / \mathrm{pi}\right), \mathrm{n}, 1, \mathrm{k}\right]\right]^{\prime}$ e a soma $H=\sum_{k=1}^{n}\left(b_{k} \operatorname{sen} \frac{k \pi x}{L}\right)$ foi definida com o comando "h=Soma[Sequência[ (1/pi)* Integral[f*sen((n* pi*x)/pi), -pi, pi]* $\left.\operatorname{sen}\left(\left(\mathrm{n}^{*} \mathrm{pi}{ }^{*} \mathrm{x}\right) / \mathrm{pi}\right), \mathrm{n}, 1, \mathrm{k}\right]$ ]", como na Figura 7.

Por fim, $F(x)=a_{0}+\sum_{k=1}^{n}\left(a_{k} \cos \frac{k \pi x}{L}+b_{k} \operatorname{sen} \frac{k \pi x}{L}\right)$, que corresponde à melhor aproximação da função $f$ por funções trigonométricas, foi definida com o comando: 


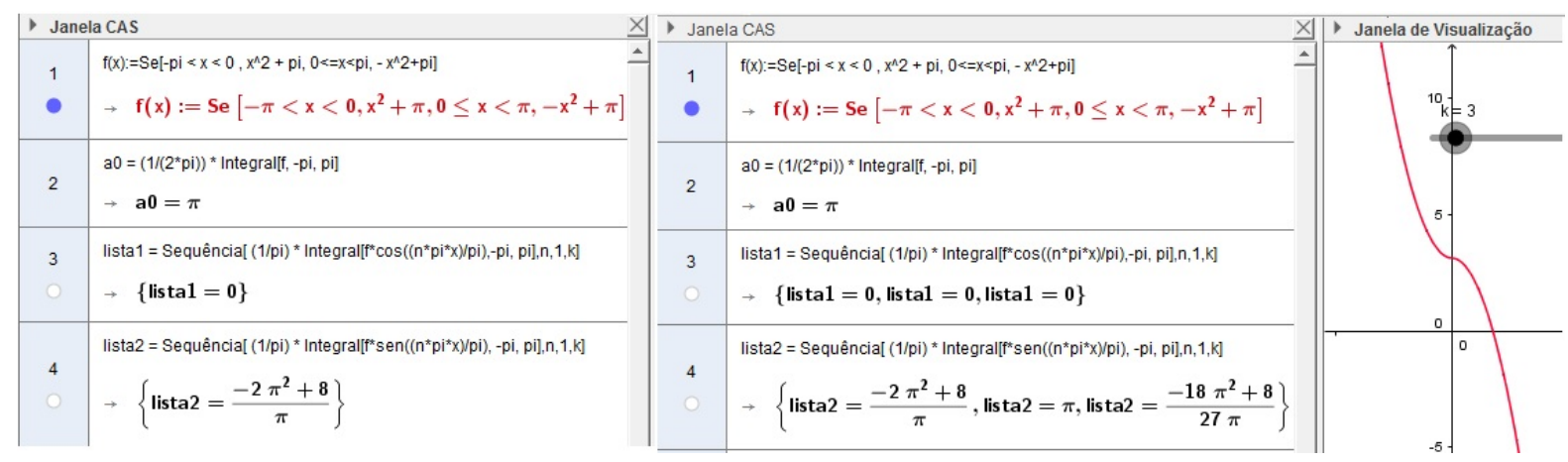

Figura 6: Definição dos coeficientes de Fourier para $k=1$ e $k=3$.

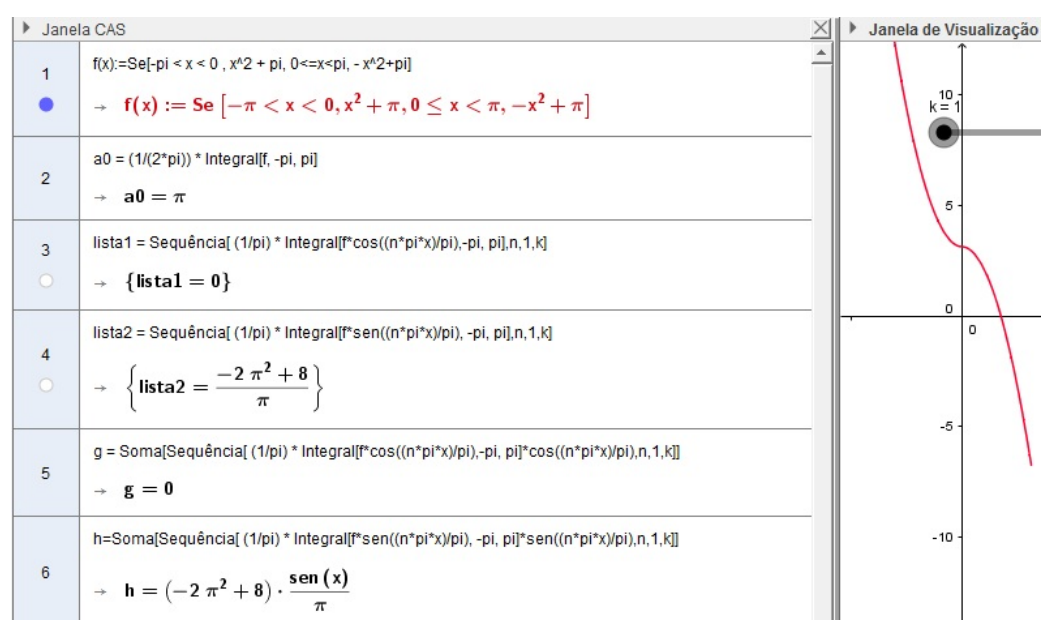

Figura 7: Definição dos termos da função $F$. 


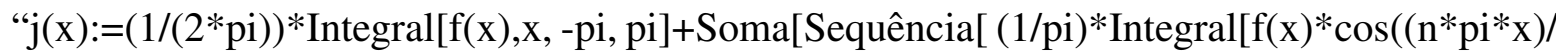
pi), $\mathrm{x},-$ pi, pi] $\left.\left.{ }^{*} \cos \left(\left(\mathrm{n}^{*} \mathrm{pi}^{*} \mathrm{x}\right) / \mathrm{pi}\right), \mathrm{n}, 1, \mathrm{k}\right]\right]+\operatorname{Soma}\left[\operatorname{Sequência[}(1 / \mathrm{pi})^{*} \operatorname{Integral}\left[\mathrm{f}(\mathrm{x}) * \operatorname{sen}\left(\left(\mathrm{n}^{*} \mathrm{pi}{ }^{*} \mathrm{x}\right) / \mathrm{pi}\right), \mathrm{x}\right.\right.$, -pi, pi]*sen $\left.\left.\left(\left(\mathrm{n}^{*} \mathrm{pi}^{*} \mathrm{x}\right) / \mathrm{pi}\right), \mathrm{n}, 1, \mathrm{k}\right]\right]^{\prime}$, que corresponde a unir os passos feitos anteriormente com o objetivo de melhorar a compreensão de cada parte que compõem o polinômio trigonométrico. Na Figura 8, pode-se observar o polinômio trigonométrico com grau 2, definido para $k=2$.

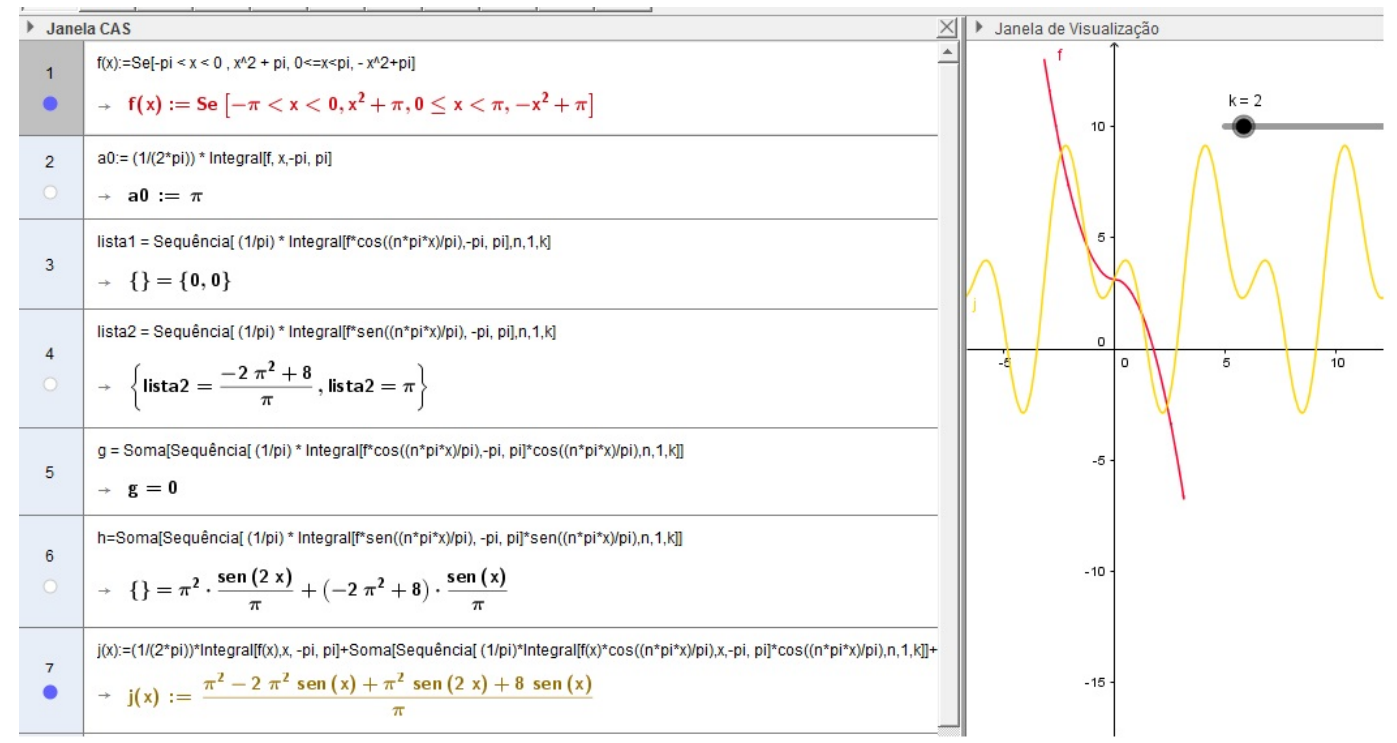

Figura 8: Aproximação de $f$ para $k=2$.

O número de parcelas do polinômio trigonométrico definido para aproximar $f$ depende do controle deslizante $k$ e a medida que $k$ aumenta, melhor é a aproximação obtida. Veja na Figura Figura 9 o polinômio obtido para $k=15$.

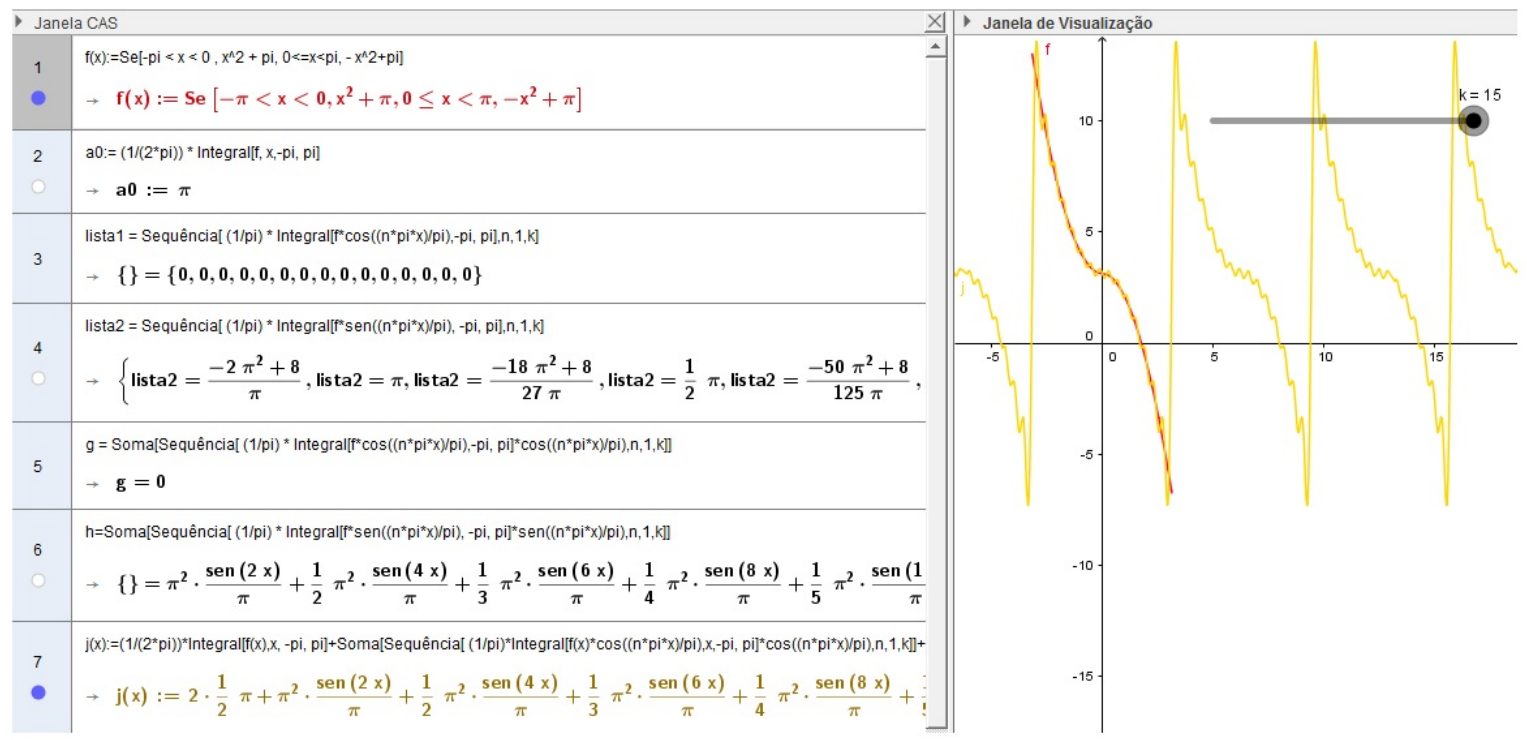

Figura 9: Aproximação de $f$ para $k=15$. 


\section{Conclusão}

Aproximar funções reais no espaço ortogonal $W$, mostrado neste trabalho, é um caminho para se obter a Séries de Fourier dessas funções. A escolha desse caminho para a obtenção das somas parciais da Série de Fourier teve por objetivo aplicar os importantes conceitos de Álgebra Linear, como projeções ortogonais e processo de mínimos quadrados. Por ser uma teoria bastante presente na resolução de equações diferenciais, as Série de Fourier merecem atenção especial. Por isso, acredita-se que associar esse estudo com a utilização de softwares de fácil utilização e construção das funções possibilita uma melhor compreensão das parcelas que envolvem essa soma e da função como um todo. Em especial, o software GeoGebra foi escolhido por apresentar as características listadas anteriormente, além de possuir o efeito dinâmico através do controle deslizante que possibilita fazer análises e comparações. O exemplo de aproximação construído no GeoGebra ilustra bem quanto maior for grau do polinômio, melhor será a aproximação obtida. Quando o número de parcelas do polinômio aproximador for infinito, ter-se-á a série de Fourier da função aproximada e, nesse caso, o espaço ortogonal utilizado para fazer a projeção ortogonal inicial terá dimensão infinita.

\section{Referências Bibliográficas}

ANTON, H.; RORRES, C. Álgebra linear com aplicações. 8. ed. Porto Alegre: Bookman, 2001.

GAMA, J. P. F; SANTANA, F. T. Aproximação de funções por polinômios trigonométricos e aplicação em sistemas mecânicos utilizando o GeoGebra. In: ENCONTRO REGIONAL DE MATEMÁTICA APLICADA E COMPUTACIONAL, 2017, Bauru. Caderno de trabalhos completos e resumos. Bauru: Unesp, Faculdade de Ciências, 2017, p. 453-455.

GEOGEBRA. O que é o GeoGebra. Disponível em: < http://www.geogebra.org/about $>$. Acesso em: 29 jul. 2017.

HOHENWARTER, M.; HOHENWARTER, J. Ajuda GeoGebra: manual oficial da versão 3.2. Disponível em: <https://app.geogebra.org/help/docupt_PT.pdf>. Acesso em: 29 jul. 2017.

MANSO, W. B; SANTANA, F. T. O Processo de mínimos quadrados aplicado no ajuste de dados com o uso do software GeoGebra. In: CONGRESSO BRASILEIRO DO GEOGEBRA, 1., 2016, Natal. Anais... Natal, RN, p. 1-6.

RAO, S. S. Vibrações mecânicas. 4. ed. São Paulo: Pearson Prentice Hall, 2008.

TRINDADE, D.; GREGÓRIO, R. Tutorial janela CAS. Disponível em: $<$ https://www.passeidireto.com/arquivo/18235763/tutorial-janela-cas-geogebra $>$. Acesso em: 29 jul. de 2017.

ZILL, D. G.; CULLEN, M. R. Equações diferenciais. São Paulo: Makron Books, 2007. v. 2.

Artigo recebido em jun. 2017 e aceito em out. 2017.

GAMA, J. P. de F.; SANTANA, F. T. Utilização do software GeoGebra para aproximar funções através de mínimos quadrados. C.Q.D.- Revista Eletrônica 